\title{
Proposal of a New Clinical Method for removal of Button Batteries and other Ferrous Material from the External Auditory Ear Canal and Nasal Cavity using a Fine Magnet Probe. \\ Din W, Kelly G, Liu CSY \\ Department of ENT, Leeds Teaching Hospitals NHS Trust
}

\section{Introduction}

- Removal of batteries from the external auditory canal (EAC) or nasal cavity is an ENT emergency.

- This should be done immediately at time of presentation, but can be especially challenging in children.

- Complications from impacted button batteries include nasal septal perforation with saddle nose deformity, bleeding, infection and liquefactive necrosis of the EAC with a malignant otitis externa type presentation.

- We previously used a magnetic probe in the form of a nasogastric tube fixation magnetic bridle,

- This worked reasonably effectively in removing a button battery from a child's ear, but was not perfect due to cost and design limitations.

- Development of a fine magnetic probe is proposed for use acutely and in ENT clinics for the removal of ferrous foreign bodies.

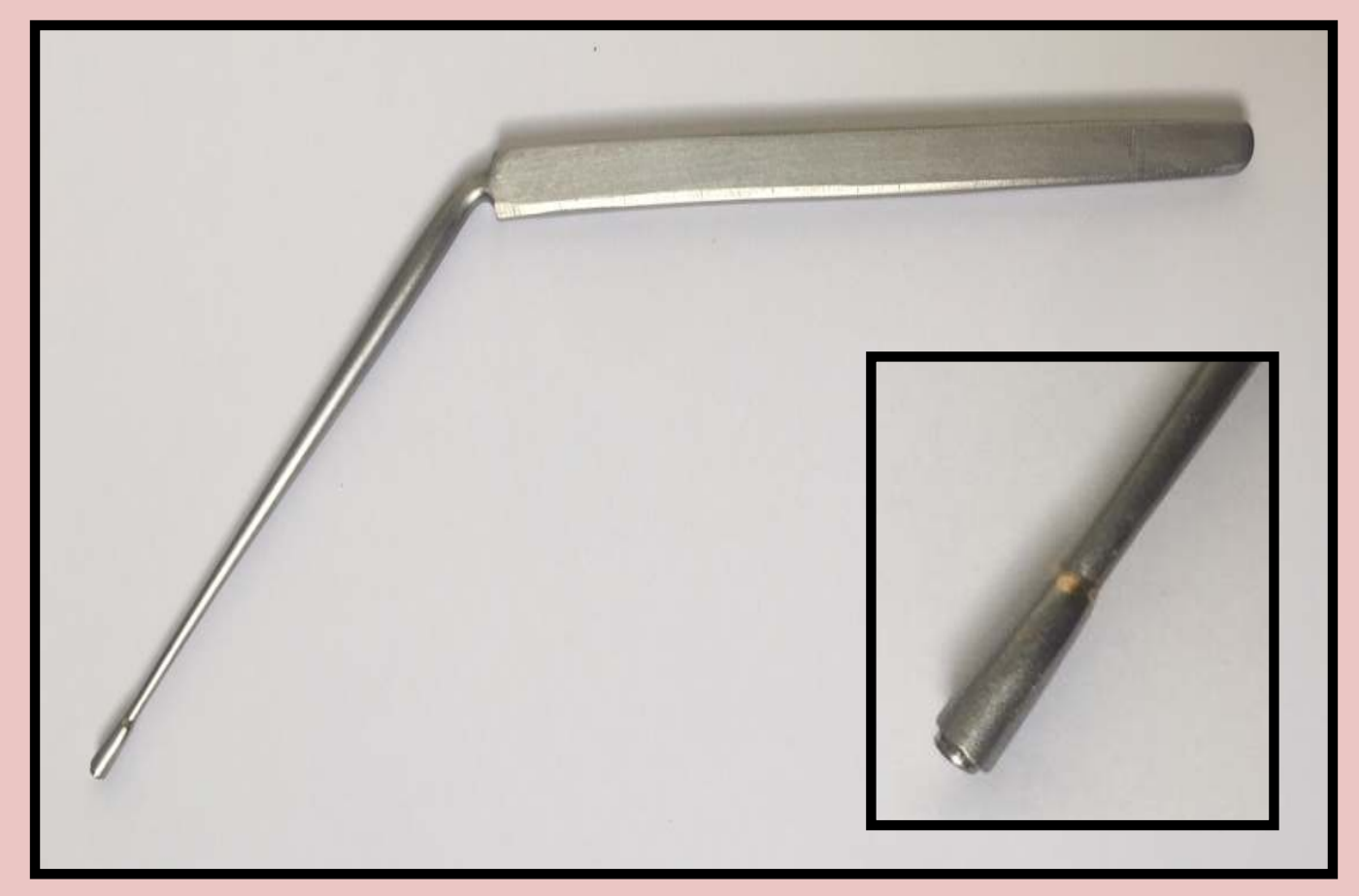

Fig 1. Steel fine magnet probe prototype with magnified tip end

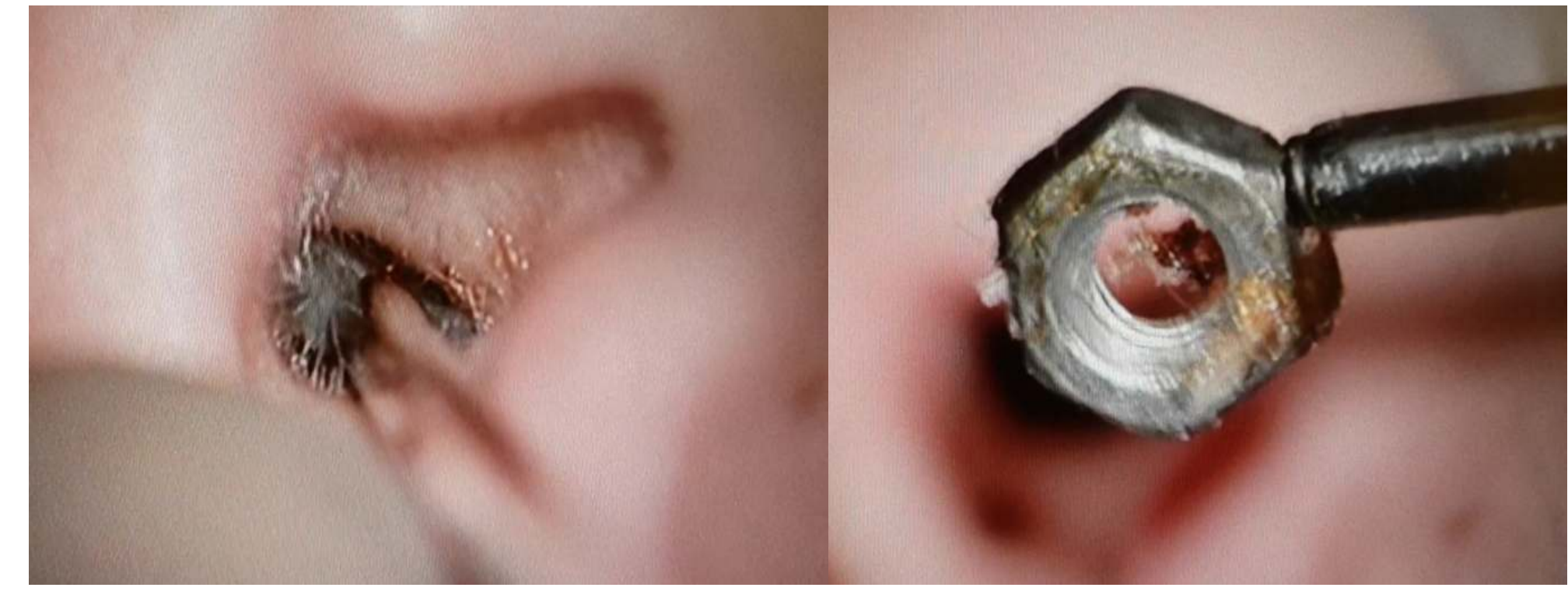

Fig 2. Retrieval of Ferrous object from 10-year-old male using early fine magnet probe prototype.

\section{Method}

- We trialled the magnetic probe prototype against a nasogastric tube fixation magnetic bridle on EAC and nasal cavity models, comparing speed of foreign body retrieval

- Retrieval was attempted by 5 senior house officers using both instruments.

- Production cost was also compared.

- The magnetic probe was then used in retrieval of a foreign body from a 10 -year-old male's EAC.

\section{Results}

- In all $5(100 \%)$ of subjects, removal of ferrous material was quicker from both EAC and nasal cavity models.

- Cost of production of the magnetic probe was $f 140$ cheaper than a nasogastric tube fixation magnetic bridle, with further cost efficiency expected in mass production.

- Removal of ferrous matter from the ear of the 10-year old boy was done in 2 minutes with no trauma to the EAC.

\section{Conclusion}

- The developed instrument had the following qualities: disposable, inexpensive, fine enough for easy access to the EAC with adequate co-axial vision and magnetically strong enough to hold button batteries or equivalent metallic bodies for prompt removal.

- The widespread use of a fine magnet probe is proposed in ENT clinics and in an acute settings for removal of ferrous foreign bodies . 